\title{
New Ways to Disseminate the US National Notifiable Disease Provisional Data
}

\author{
Anna Grigoryan* \\ CDC, Atlanta, GA, USA
}

\section{Objective}

The purpose of this study was to identify ideas for an enhanced dissemination of the US National Notifiable Diseases Surveillance System (NNDSS) provisional data.

\section{Introduction}

The NNDSS is a nationwide collaboration that enables all levels of public health (local, state, territorial, federal and international) to monitor, control, and prevent the occurrence and spread of statereportable and nationally notifiable diseases and conditions. The NNDSS data are a critical source of information for monitoring disease trends, effectiveness of prevention and control programs, and policy development. To provide timely NNDSS data, state and territorial health departments voluntarily report notifiable disease incidence data to CDC when they become aware of these cases and as per recommended national notification timeframes. These provisional data are published each week in Morbidity and Mortality Weekly Report (MMWR). Great strides have been made exploring and exploiting new and different sources of disease surveillance data and developing robust statistical methods for analyzing the collected data (1). However, there have been fewer efforts in the area of online dissemination of surveillance data. Appropriate dissemination of surveillance data is important to maximize the utility of collected data (2).

\section{Methods}

The author conducted a search of all US State Health Department websites looking for on-line data display tables and tools for either "reportable" or "notifiable" diseases. In addition, the scope of the search of websites was expanded to include notifiable diseases of several countries, organizations and institutions. Several articles relevant to the topic of the study were reviewed as well. There were few examples of ways to display data that may be more effective and useful to data users than the current static NNDSS data display. Particular emphasis was placed on the display of reportable or notifiable disease provisional data. Provisional NNDSS data in MMWR Table II are a running count of selected nationally notifiable infectious disease cases that have not been adjusted for variations in reporting procedures across different states or for delays in reporting. As a result, provisional data are subject to change based on the outcome of further case investigation.

\section{Results}

Current NNDSS provisional data display problems can be summarized as an overall unsatisfactory user experience due to the following factors:

- Information: static MMWR table format, cluttered presentation, data not easily accessible for further analysis;

- Technology: not user-friendly;

- Location: information separated into different places (NNDSS website, different notifiable diseases websites maintained by different CDC Programs, etc.); and

- Service: not catered to users individual needs and overall not helpful.
As a result of this study the author proposes enhancement of the current notifiable disease data display from a static format to a more interactive dashboard design. Proposed NNDSS dashboard can help users to overcome information overload, saves users time through quick access to a database and provides opportunity to present data in a more effective way. Users from states can also compare their epidemiological findings with other states in order to plan and collaborate on important public health issues. Several currently nonexisting display options need to be added as multiple area comparisons to facilitate geographical comparison across different US States, etc.

\section{Conclusions}

Advances in infectious disease informatics research in recent years has allowed significant improvements not only in data collection, sharing, reporting, and analyzing, but also in data dissemination and visualization maximizing the data usage. The findings of this study are subject to a few limitations: 1) only English public websites and/ or databases were reviewed based on the search term strategy; 2) this review focused on the dissemination of surveillance data, and has not reviewed the underlying surveillance systems.

Dissemination of the US national notifiable disease provisional data using interactive dashboard design can take advantage of what technology can offer nowadays.

\section{Keywords}

Public Health Surveillance; Data Display; Public Health Informatics; United States

\section{References}

1. Teutsch SM, Churchill RE, eds. Principles and practice of public health surveillance. 2nd edition. Oxford University Press; 2000

2. Cheng CK, Lau EH, Ip DK, et al. A profile of the online dissemination of national influenza surveillance data. BMC Public Health. 2009; 9: 339

*Anna Grigoryan
E-mail: Ffg7@cdc.gov and reproduction in any medium, provided the original work is properly cited. 